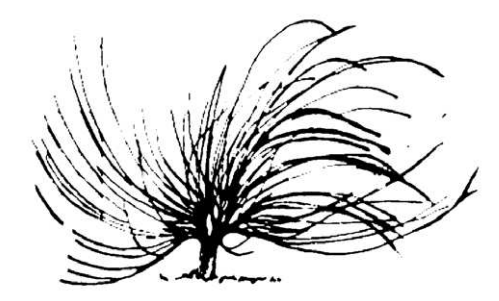

\title{
La práctica del judo como una modulación del autoconcepto, la autoestima y el rendimiento escolar de los estudiantes de primer ciclo de educación primaria
}

\author{
Marco Alexandre da Silva Batistal \\ Escuela Superior de Educación, Portugal \\ Torres Novas, Portugal \\ marcobatist@gmail.com \\ Sixto Cubo Delgado ${ }^{2}$ \\ Universidad de Extremadura, España \\ Badajoz, España \\ sixto@unex.es
}

\begin{abstract}
Resumen
Este estudio ha tenido como objetivo relacionar la práctica del judo en la formación de autoconcepto, autoestima y rendimiento escolar en niños del primer ciclo de primaria. Hemos trabajado con una muestra de 531 alumnos portugueses, con una media de 9,13 años, variando un mínimo de 8 y un máximo de 10 años. De esta muestra, 394 alumnos (74\%) practican por lo menos una actividad física formal de carácter extracurricular y 96 alumnos $(24 \%)$ practican judo, con una experiencia entre 9 meses y 5 años. Como instrumento de recogida de datos se utilizó la Escala de Autoconcepto de SusanHarter validada para la población portuguesa (Martins, Peixoto, Mata y Monteiro,
\end{abstract}

Recibido: 18 de febrero de 2013 - Aprobado: 11 de octubre de 2013

1 Doctorado en Ciencias de la Educación, Máster en Psicología del Deporte y una Licenciatura en Educación Física. Trabaja actualmente en la Escuela Superior de Educación de Torres Novas, en Portugal.

2 Doctorado en Ciencias de la Educación y una licenciatura en Psicología. Actualmente trabaja en la Universidad de Extremadura en la Facultad de Educación Badajoz, en España. 
1995). También se han registrado los rendimientos escolares. Se crearon grupos de discusión con entrenadores de judo y encargados de educación con la intención de averiguar opiniones y argumentos sobre la práctica del judo y su importancia y relación con el autoconcepto, autoestima y rendimiento escolar. Aparentemente los resultados revelan que la práctica del judo por los alumnos promueve una evolución significativa en la formación de autoconcepto y rendimiento escolar, así como sugiere una tendencia evolutiva positiva en la formación de la autoestima de los alumnos practicantes de la modalidad.

Palabras clave: bienestar psicológico, autoconcepto, autoestima, judo, rendimiento escolar.

\begin{abstract}
This study aimed at relating the general practice of judo to the development of self-concept, self-esteem, and school performance in children of the first cycle of primary school education. We worked with a sample of 531 Portuguese students, with an age mean of 9.13 years old, ranging from a minimum of 8 and a maximum of 10 years old. Out of this sample, 394 students (74\%) do at least one formal extracurricular physical activity, and 96 students (24\%) practice judo with an experience ranging between 9 months and five years. As an instrument for data collection, we used the Scale of Self-concept by Susan Harter validated for Portuguese population (Martins, Peixoto, Mata, \& Monteiro, 1995). Also, school performances have been recorded. Discussion groups with judo coaches and parents were held in order to find out opinions and arguments about the practice of judo and its importance and connection to self-concept, self-esteem, and school performance. Apparently, the results show that students' practice of judo promotes a significant evolution in the development of self-concept and academic achievement, and also they suggest an evolutionary trend in positive self-esteem in students who practice judo.
\end{abstract}

Keywords: psychological well-being, self-concept, self-esteem, judo, school performance 


\section{Introducción}

$\mathrm{L}$

a Organización de las Naciones Unidas para la Educación, la Ciencia y la Cultura - UNESCO reconoció la pedagogía y el mación del ser humano.

El judo, como método pedagógico, interviene en el dominio cognitivo, social y físico-motor, lo que es un excelente medio de desenvolvimiento motor del niño, contribuyendo para su educación y formación, ya que promueve actividades saludables en un ambiente agradable, promocionando el bienestar físico y psicológico (Batista y Cubo, 2011). El bienestar psicológico reúne una serie de formas específicas, especialmente la autoestima, componente relacionada con el autoconcepto, lo que el judo, como actividad física, potencia el desarrollo y promoción de ambos.

No solo por las posibles relaciones con el aprovechamiento/rendimiento escolar, como por sus potencialidades para promover la salud y el bienestar, la sociomotricidad y también como fomentador de la creación/ mantenimiento de hábitos de vida saludables y de higiene personal.

La adhesión a la práctica de una actividad física, en particular el judo, es un componente de un proceso complejo que envuelve el desarrollo, el aprendizaje y la asimilación de competencias, valores, normas, autopercepciones, identidades y papeles proporcionados por variables diferentes del encuadramiento familiar y de las relaciones sociales, como el grupo sociodemográfico, la profesión, la educación y el área de residencia (Castarlenas y Molina, 2002 y Carratalá, 2012). Estos factores ejercen influencia en la práctica de la actividad física de los niños y de los adolescentes en su tiempo libre (Yang, Telama y Laakso, 1996 y Hellstedt, 1995), así como Musitu, Román y Gutiérrez (1996), refieren que el medio ambiente familiar y escolar de los alumnos es un factor importante en el desarrollo del autoconcepto de los jóvenes judocas o deportistas.

\section{Marco Teórico}

JigoroKano, fundador del judo, que se define como el uso más eficiente de la mente y el cuerpo para el beneficio y bienestar de los demás. El buen uso de la mente y el cuerpo, su energía para un buen desarrollo personal, por lo tanto, beneficiar al mundo (Schuler, 2007). 
La adhesión a la actividad física, incluyendo judo, es un componente de un proceso complejo que implica el desarrollo, el aprendizaje y la asimilación de conocimientos, los valores, las normas, las percepciones de uno mismo, las identidades y los papeles proporcionados por diferentes variables y el entorno familiar, participación social, como el grupo socio-demográfico, la profesión, la educación y la zona de residencia, que pueden ser posibles factores que influyen en los niveles de actividad física de niños y adolescentes en su tiempo libre (Yang, Telama y Laakso, 1996).

Autores como Cocke (2002), Tremblay, Inman y Willms (2000) Dwyer et al., (1983) y Shephard (1997) afirman que la parte de jóvenes que participan en la actividad física regular tienden a mostrar mejores atributos como aumento de la actividad cerebral y su nutrición, los niveles de concentración y energía, cambios en la composición corporal que afecta a la autoestima y el comportamiento de la persona por lo positivo, lo que puede dar un mayor apoyo para el aprendizaje cognitivo.

Parece claro también que la asociación de la actividad física con la autoestima y la autoeficacia (McAuley y Rudolph, 1995 en Mota, 1998), en el bienestar emocional parece ser evidencia de un efecto positivo de la actividad física en la reducción de síntomas depresivos y de ansiedad (Brown, Wang \& Ward, 1995 scouts en 1999). Según McAuley y Rudolph (1995 en Mota, 1998), el ejercicio contribuye a la integridad del complejo vascular cerebral, aumentando el transporte de oxígeno en el cerebro, síntesis y degradación de neurotransmisores.

Neto (1994) afirma que el deporte es una actividad en sus supuestos, que debe incluir la promoción de los valores humanos, los principios de solidaridad y cooperación social y cultural entre sus grupos de interés, las cualidades exaltadas en la práctica del judo.

El reconocimiento de la pedagogía y el método de judo se efectuará mediante Organización de las Naciones Unidas para la Educación, la Ciencia y la Cultura - UNESCO como utilitario deportivo mundial en la formación del ser humano y una actividad cuyo espectro de enfoque en los grupos de edad infantil es muy amplia, tiene un papel preponderante en la formación integral de sus practicantes, lo que les permite transferir el contexto deportivo para su contexto socio-cultural específico.

Judo contribuye al desarrollo social y emocional de los niños, ya que su práctica está llena de valores morales importantes. Un niño que ha practicado judo, aprende a ser disciplinado, a confiar en sí mismo, para adquirir el autocontrol y la autoestima, para adaptarse a las nuevas 
situaciones y problemas, cooperar, comprometerse y resistir (Castarlenas y Molina, 2002 ). El judo, como un método pedagógico, tiene un ámbito de intervención en el desarrollo cognitivo, social y físico-motor, que creemos que es un excelente medio para el desarrollo motor del niño, lo que contribuye a una buena educación y formación de ella, ya que promueve actividades saludables en un ambiente agradable, la promoción de un bienestar físico y psicológico. El bienestar psicológico reúne una serie de formas específicas, como componentes de la autoestima en relación con el autoconcepto, que creemos judo como la actividad física, fomenta el desarrollo y la promoción de ambos.

Según Nash (1970), la imagen del cuerpo y su esquema corporal son términos usados para describir los componentes relacionados con el cuerpo que se incluyen en el concepto de sí mismo. Sastre (1999), Vasconcelos et al. (2004) se refieren entre otras cosas al cuerpo físico y la apariencia física como un factor de bienestar. El ejercicio como un medio para la promoción de la salud es un principio aceptado, y un tiempo a esta parte incluye la noción de que el bienestar psicológico está vinculado a las tasas de participación en la actividad física por los individuos (Biddle y Mutrie, 2001).

Entre las variables que se utilizan para evaluar el bienestar psicológico, la autoestima parece ser la que aporta un mayor consenso (Sonstroem, 1984, 1997, Fox, 2000). Biddle y Mutrie (2001) afirman que esta es la variable más importante para evaluar el bienestar. En opinión de Lucas, Diener y Suh (1996), la autoestima está fuertemente asociada con el bienestar psicológico. El bienestar psicológico se puede entender en al menos dos dimensiones: cognitiva (satisfacción) y afectivo (felicidad). Estas dos dimensiones abarcan una serie de conceptos tales como la competencia escolar, la aceptación social, competencia atlética, la apariencia física, la autoeficacia, la actitud de comportamiento, que son componentes de la formación del concepto de sí mismo. Vasconcelos Raposo, Gonçalves y Teixeira (2004) ponen de relieve el hecho de que la satisfacción del cuerpo es un factor que tiende a ser pasado por alto por los estudiosos en el campo de la investigación científica del bienestar psicológico.

Simon (2005), quienes reportaron la depreciación atribuibles a la participación en el factor de actividad física extracurricular como algo beneficioso para el rendimiento académico antes del siglo XX, en última instancia llegó a la conclusión de que esa participación incide positivamente 
en el rendimiento académico y que los estudiantes que participaron en actividades extracurriculares tienen una mayor percepción de las dimensiones de competencia en la escuela y la aceptación social.

En su opinión, Samulsky (2002) señala que el tipo de ejercicio aeróbico ha demostrado ser un medio eficaz para obtener el bienestar psicológico, con efectos positivos sobre el estrés, estado de ánimo y autoconcepto.

No sólo por sus posibles relaciones con el rendimiento de uso / de la escuela, y por su potencial para promover la salud y el bienestar, la socio motricidad, sino también como desarrolladores para crear / mantener hábitos de vida saludables y de higiene personal, esta actividad y sus ganancias en la formación de la personalidad del individuo, deben ser objeto de enfoque obligatorio en el currículum de la educación física en el sistema educativo portugués, al igual que otros países europeos en los que asume un lugar destacado en Francia.

Puso de relieve los beneficios de las actividades físicas y deportivas, se destaca el bienestar psicológico y los factores que contribuyen a este estado, donde el concepto de sí mismo es de gran importancia, sobre todo en la dependencia psicosomática en el diseño del propio cuerpo y la prestación posterior el rendimiento escolar.

\section{Método}

Con este trabajo pretendemos relacionar la práctica del judo con la formación de autoconcepto, autoestima y rendimiento escolar en niños del primer ciclo de primaria.

Los objetivos específicos de este estudio son:

1. Verificar la influencia de la práctica del judo en el autoconcepto, autoestima y rendimiento escolar de judocas.

2. Identificar el perfil de autoconcepto, autoestima y rendimiento escolar de judocas y establecer comparaciones con no practicantes y practicantes de otras modalidades.

3. Encontrar más-valías pedagógicas de la modalidad de judo en la formación del autoconcepto de niños del primer ciclo de enseñanza básica, en las áreas de competencia escolar, aceptación 
social, competencia atlética, aspecto físico, comportamiento, autoestima global.

4. Relacionar más-valías pedagógicas de la modalidad de judo con el rendimiento escolar global de los alumnos.

En esta investigación, las variables en estudio son el autoconcepto en sus dimensiones constituyentes (competencia escolar, aceptación social, competencia atlética, aspecto físico y comportamiento); la autoestima y el rendimiento escolar, que fueron analizadas en relación con las variables: volumen capital, formación de los padres, número de veces de práctica semanal de judo además de las clases de educación física en la escuela, graduaciones entre cinturones y el tiempo total de práctica de la modalidad.

Trabajamos con una muestra de 531 alumnos de Santarém, Torres Novas, Entroncamento y Tomar (Portugal), entre 2010 y 2011, de los cuales $295(55,6 \%)$ de género masculino y $236(44,4 \%)$ de género femenino, con una media de 9,13 años, variando un mínimo de 8 años y un máximo de 10 años.

De esta muestra, 394 alumnos (74\%) practican por lo menos una actividad física formal de carácter extra-curricular y 96 alumnos (24\%) practican judo, con una experiencia entre 9 meses y 5 años y graduaciones entre cinturón blanco y cinturón verde.

Como instrumento de recogida de datos, se utilizó la Escala de Autoconcepto de SusanHarter validada para la población portuguesa (Martins, Peixoto, Mata y Monteiro, 1995), en donde procedemos a un análisis descriptivo e inferencial de los datos de manera para confrontar los niveles medios de autoconcepto, en la áreas de competencia escolar, aceptación social, competencia atlética, aspecto físico, comportamiento y autoestima global. También se han registrado los rendimientos escolares de los alumnos en estudio en matemáticas, lengua portuguesa y estudio del medio.

Se crearon grupos de debate con seis entrenadores de judo y ocho encargados de educación con la intención de averiguar opiniones y argumentos sobre la práctica del judo, su importancia y su relación con el autoconcepto, autoestima y rendimiento escolar.

Concretamente, nuestro trabajo se centra en una investigación esencialmente cuantitativa de base correlacional y causal, con recurso a datos de carácter cuantitativo y cualitativo, de carácter descriptivo, de 
modo que no interfiere con la realidad, apenas se limita a analizarla, sin aplicar estímulos en la muestra en estudio.

\section{Resultados}

Nuestro trabajo demostró que los alumnos que proceden de familias con volumen de capital menor, en otras palabras, de status socioeconómico menor, demuestran una preferencia mucho mayor por modalidades colectivas, solo por la práctica de educación física o no practican ninguna actividad física formal, resultados que están de acuerdo con Bourdieu (1979). Los alumnos que optan por la práctica del judo proceden de una base familiar variada y existe un equilibrio en cuanto al volumen de capital de la familia de origen. Entre los padres de los alumnos que practican judo se registraron cerca de 55\% de licenciados, porcentaje que es mayor entre las madres $(60 \%)$ que entre los padres (50\%). Rosa (2008a) dice que el judo ocupa el primer lugar en la clasificación con el mayor número de practicantes de artes marciales y deportes de combate, afirma que son modalidades esencialmente dominadas por el sexo masculino con capitales escolares elevados. De acuerdo con los resultados de nuestro trabajo, nos encontramos aparentemente en un proceso de inversión en el judo, en relación con el encuadramiento descrito por Bourdieu (1979), que observa las actividades deportivas opcionales de cada clase social, reveló que los elementos de niveles sociales y económicos más elevados optaban por actividades individuales, sin contacto corporal, mientras que los de niveles sociales y económicos menos elevados, eran atraídos por las actividades colectivas y de contacto corporal.

Aparentemente, la práctica del judo por los alumnos promueve una evolución significativa en la formación de autoconcepto y rendimiento escolar, así como sugiere una tendencia evolutiva positiva en la formación de la autoestima de los alumnos practicantes de la modalidad. 


\section{Figura 1: Promedios del autoconcepto y la autoestima como una función de la variable de la práctica de judo frente a otras modalidades (Batista y Cubo, 2011)}

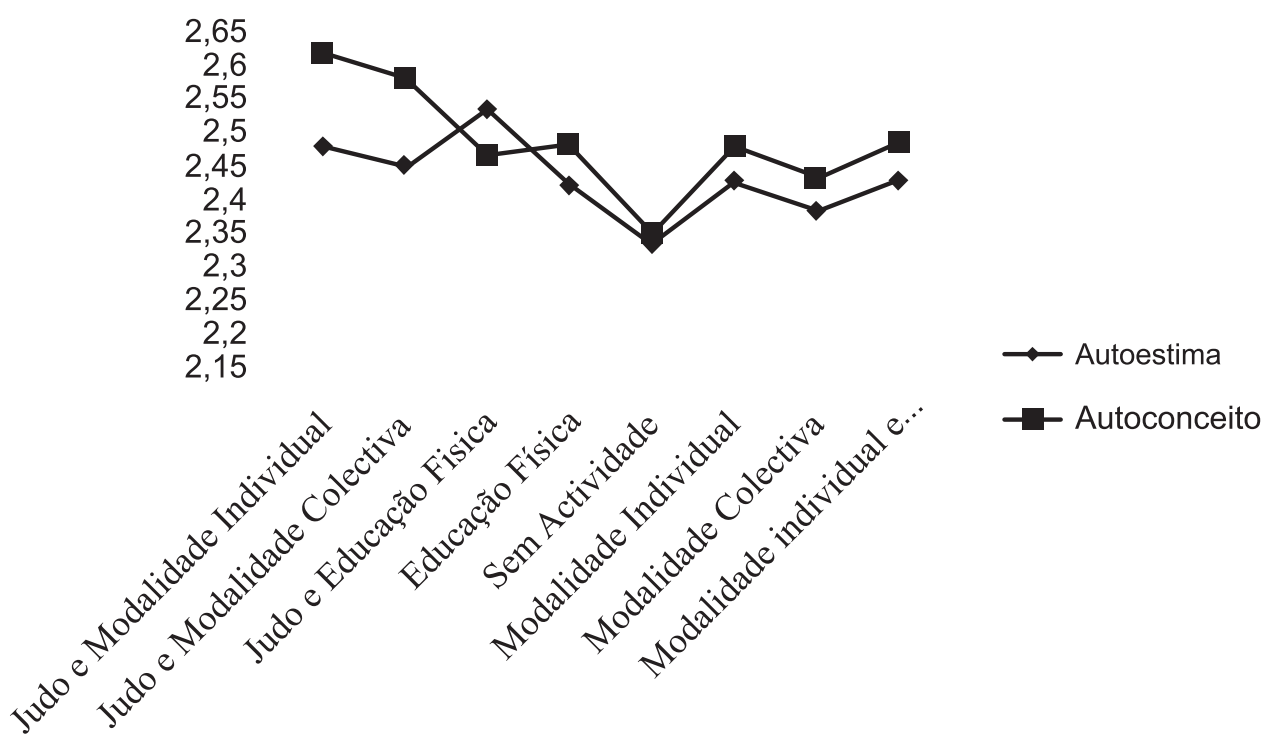

Los resultados fueron igualmente favorables a la idea de que la práctica del judo ejerce una función modeladora sobre la formación del autoconcepto, autoestima y rendimiento escolar, ya que los niveles de estas variables observadas en alumnos que sólo practican modalidades colectivas son bastante más modestos, lo que no sucede cuando añaden el judo a sus actividades. La ausencia de la práctica de cualquier actividad física y deportiva de carácter formal está relacionada con niveles de autoconcepto, autoestima y rendimiento escolar inferiores. 
Figura 2: Rendimiento promedio escolar en función a la práctica de judo frente a otras modalidades (Batista y Cubo, 2011)

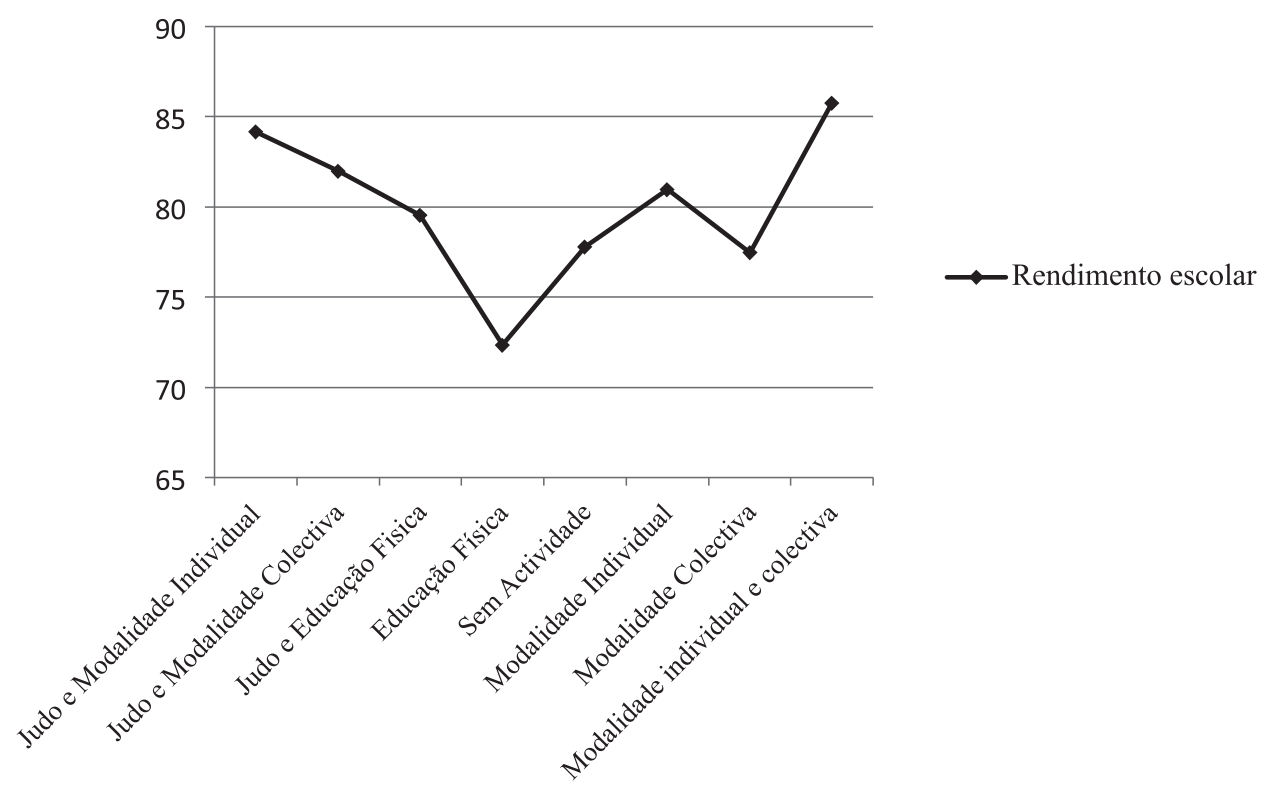

Desde el punto de vista de los responsables de educación y de los entrenadores de los alumnos que practican judo, esta debería ser una modalidad enfocada como unidad curricular obligatoria en los programas de educación física dentro de los diferentes ciclos de enseñanza, de acuerdo con los grandes valores educativos, formativos y pedagógicos que caracterizan esta modalidad, resultado de una riqueza cultural que procede y se mantiene desde su origen.

Los resultados refuerzan la opinión de Castarlenas y Molina (2002) que afirma que la práctica del judo contribuye, de una manera importante, para el crecimiento del niño tanto desde el punto de vista motor e intelectual como moral. Se encuentran también de acuerdo con Marivoet (2001), que refiere que la práctica deportiva en la escuela funciona como el primer lugar donde la mayoría de los niños tienen su primera experiencia deportiva, debiendo asegurar la escuela como una práctica variada y continua de educación física y deportes escolares.

La formación deportiva ecléctica de los alumnos y la posibilidad de defensa personal han sido también realzadas, revelándose esta modalidad como un factor motivacional extrínseco que podrá mejorar el rendimiento y la actuación de los alumnos. Estos resultados no están en absoluto de acuerdo con los revelados por Rosa (2008b), que señala 
las razones indicadas como importantes en la perspectiva de los participantes de artes marciales, el bien-estar físico, psicológico y el placer que suscitan en la práctica. La defensa personal y la competición son los motivos menos referidos por los practicantes.

Para los encargados de educación y entrenadores, frente a las distintas dimensiones que constituyen el autoconcepto, autoestima y rendimiento escolar, la práctica del judo sugiere la promoción de un buen desarrollo de las capacidades físicas condicionales y coordinativas, un buen desarrollo morfológico y psicomotor, en la medida que modela las capacidades atencionales y posturales del alumno, favorece en el aprendizaje y la capacidad de discriminación de contenidos y objetivos, así como de la concentración y raciocinio, corroborando de esta forma las perspectivas de Queiroz y Gomes (s.f.),Delgado (2005) y Oliveira (2008). Las capacidades de gestión del tiempo en función de sus obligaciones y responsabilidades parecen ser favorecidas, promoviendo mayor confianza de actuación, sea en situaciones familiares y/o desconocidas, que deriva de una mejoría de su autoconocimiento y autocontrol, tal como es referido por Castarlenas y Molina (2002) o por Delgado (2005). Por lo tanto, interviene en la autoconfianza y en la aceptación social por sus compañeros o líderes, factores que interfieren en la autoestima.Por lo tanto, los responsables de educación y los entrenadores creen que el judo aparece como un enfoque disciplinario dentro del contexto escolar o como modalidad deportiva, con gran valor pedagógico y de socialización de forma que modela el perfil de comportamiento del individuo, en el dominio de los valores éticos y morales, fundamentado en la disciplina y cumplimiento de las reglas, factores que contribuyen para un buen rendimiento escolar, resultado de la formación y evolución del autoconcepto y de una autoestima adecuada. Esta conclusión de los grupos de debate, está en paralelo con la opinión de Neto (1994) y Bento $(1995,2004)$, que refieren que el deporte es una actividad que debe incluir en sus presupuestos la promoción de los valores humanos, los principios de solidaridad y la cooperación social y cultural entre sus participantes, entendiéndose como un bien pedagógico.

Además de las relaciones con el rendimiento escolar, como por su potencial para promover la salud y el bienestar, la sociomotricidad, pero también para promover la creación/manutención de costumbres saludables y de higiene personal permite un estado superior de bienestar psicológico. El bienestar psicológico reúne una serie de formas 
específicas, particularmente, la autoestima componente relacionada con el autoconcepto y creemos que el judo, como actividad física, potencia el desarrollo y promoción de ambos. Brown, Wang y Ward (1995, en Esculcas, 1999) mantienen la idea de que, relativamente al bienestar emocional, parece haber evidencias de un efecto positivo de la actividad física en la reducción de los síntomas depresivos y de ansiedad.

\section{Discusión}

Del gran objetivo de este trabajo, donde hemos buscado relacionar la práctica del judo con la formación del autoconcepto, autoestima y rendimiento escolar en los niños del primer ciclo de enseñanza básica, por los resultados obtenidos, se ha verificado una tendencia cada vez mayor de los niveles de autoconocimiento con la evolución en la graduación. Por lo tanto, los alumnos con graduaciones de cinturones más elevadas presentan un nivel de autoconocimiento significativamente superior al de los alumnos menos graduados en esta modalidad, siendo que esta tendencia fue igualmente observada por Batista y Cubo (2009).

Tabla 1: Análisis inferencial del autoconcepto, autoestima y rendimiento escolar en función a la práctica de judo frente a otras modalidades

\begin{tabular}{|c|c|c|c|c|c|c|}
\hline $\begin{array}{c}\text { DOMINIO } \\
\text { DEPORTIVO }\end{array}$ & $\begin{array}{c}\text { Média } \\
\text { Autoconcepto }\end{array}$ & $\begin{array}{c}\text { Kruskal } \\
\text { Wallis } \\
\text { Sig. }\end{array}$ & $\begin{array}{c}\text { Média } \\
\text { Autoestima }\end{array}$ & $\begin{array}{c}\text { Kruskal } \\
\text { Wallis } \\
\text { Sig. }\end{array}$ & $\begin{array}{c}\text { Média } \\
\text { Rendimiento } \\
\text { Escolar }\end{array}$ & $\begin{array}{c}\text { Kruskal } \\
\text { Wallis } \\
\text { Sig. }\end{array}$ \\
\hline $\begin{array}{l}\text { Modalidad } \\
\text { Individual }\end{array}$ & 2,49 & \multirow{5}{*}{0,214} & 2,44 & \multirow{5}{*}{0,999} & 81,18 & \multirow{5}{*}{$0,000 * * *$} \\
\hline $\begin{array}{l}\text { Modalidad } \\
\text { Colectiva }\end{array}$ & 2,48 & & 2,44 & & 76,55 & \\
\hline $\begin{array}{l}\text { Modalidad } \\
\text { Individual e } \\
\text { Colectiva } \\
\end{array}$ & 2,51 & & 2,43 & & 85,23 & \\
\hline $\begin{array}{l}\text { Educación } \\
\text { Física }\end{array}$ & 2,48 & & 2,42 & & 72,19 & \\
\hline $\begin{array}{l}\text { Ninguna } \\
\text { Actividad }\end{array}$ & 2,36 & & 2,34 & & 78,28 & \\
\hline
\end{tabular}

*Sig. $\alpha<0,05 * *$ Sig. $\alpha<0,01 * * *$ Sig. $\alpha<0,001$

(Batista y Cubo, 2011)

Los resultados han revelado también que el aumento del número de años de práctica del judo da lugar a una evolución análoga de los niveles de autoconcepto; sin embargo, no se observó un aumento 
significativo del nivel de autoconcepto del grupo con menos experiencia frente al grupo con más experiencia, como Batista y Cubo (2009) habían observado. Es importante referir que Faustino (1994) opina que el autoconcepto se estabiliza con el aumento de la edad.

Según los entrenadores, el judo, en la formación del autoconcepto, parece sugerir una tendencia positiva en la medida en que promueve el cumplimiento de reglas y la aceptación de responsabilidades.

Equilibra y modela el comportamiento y promueve la determinación en los objetivos en que se centra, en una filosofía de vida basada en valores éticos y morales y en una perspectiva de vida activa. Favorece la aceptación social, siendo favorable a nivel de competencia escolar y atlética, identificándose con su apariencia física, desenvolviendo al mismo tiempo su autoestima.

En la muestra de judocas evaluada, los niveles de autoconcepto se mostraron elevados de una forma global, estando de acuerdo con la opinión de los responsables de educación y los entrenadores, así como mostrando una relación con los estudios de Cocke (2002), Tremblay, Inman y Willms (2000) Dwyer, Coonan, Leitch, Hetzel y Baghurst (1983), Shephard (1997), Batista y Cubo (2009), donde el colectivo juvenil que participa en actividades físicas regulares tiende a demostrar mejores características como el aumento de la actividad cerebral y respectiva nutrición, de los niveles de concentración y de energía, de las alteraciones en la composición corporal, afectando el autoconcepto y el comportamiento del individuo positivamente, lo que puede proporcionar una base mayor para el aprendizaje cognitivo.

La graduación y el total de años de práctica de judo no se revelaron aparentemente como factores significativos en la formación de la autoestima, aunque aparezcan como factores modeladores de la autoestima en un sentido creciente, a favor de los alumnos con graduación de cinturón más elevado. Sin embargo, Batista y Cubo Delgado (2009) observaron una tendencia inversa, donde los grupos más jóvenes, con menos experiencia y menor graduación, aparentemente sobrevaloran la autoestima en su autoevaluación. Según los responsables de educación y los entrenadores, la práctica del judo como influencia en la autoestima, tiende a modelar esta variable, promoviendo la autoconfianza, la concentración y el autocontrol en su comportamiento. El aspecto y la forma física de los alumnos son favorables, saliendo beneficiada su integración social. 
El rendimiento escolar de los alumnos que practican judo no depende de forma significativa de la evolución en el proceso de graduación ni de los años de práctica; sin embargo, se observa una tendencia de evolución positiva a favor del grupo de alumnos con graduación de cinturón más elevado con un total de años de práctica superior. En opinión de los responsables de educación y de los entrenadores, el judo como influencia en el rendimiento escolar, ejerce una tendencia positiva ya que surge como un factor motivacional extrínseco, obligando a una gestión del tiempo superior, promueve la concentración y la capacidad de atención basada en una actitud disciplinada, ética y moral, facilitando el aprendizaje y el desarrollo psicomotor.

La práctica del judo promueve un nivel de autoconcepto significativamente superior al de los alumnos que no practican el deporte. Como señalan Batista y Cubo Delgado (2009), también en este estudio, la práctica del judo parece favorecer el nivel de autoconocimiento de los alumnos que se inscriben en esta práctica, especialmente, si esta aparece asociada a la práctica de otra modalidad individual, aun así, bastante favorecida cuando se asocia a modalidades colectivas. La ausencia de cualquier tipo de práctica formal de actividad física tiende a bajar significativamente los valores de autoconcepto de los alumnos, tal como la práctica únicamente de modalidades colectivas ha revelado valores de autoconcepto bastante modestos.

La práctica del judo no aparece como un factor que tienda a revelar niveles de autoestima significativamente superiores comparados con alumnos que practican otra modalidad deportiva. Aunque no sean significativos, los valores más destacados han sido los registrados en el grupo de alumnos que mantienen la práctica del judo asociada a la educación física curricular, seguidos por los que asocian el judo a una modalidad individual y después los alumnos que practican judo y una modalidad colectiva, este registro idéntico a los resultados obtenidos por Batista y Cubo (2009), en la medida en que Gallahue y Ozmun (2001), Weiss (1996), Batista (1995) dicen que una autoestima alta ha sido asociada a buenos rendimientos en actividades físicas o simplemente a su práctica. Por otro lado, los alumnos que no presentan cualquier tipo de práctica deportiva formal, han demostrado el valor más reducido de autoestima en la muestra estudiada.

La práctica del judo promueve niveles de rendimiento académico significativamente superiores comparativamente a otras modalidades 
deportivas. Los resultados del rendimiento escolar son favorables a los alumnos que practican judo, en especial, cuando estos la asocian a la práctica de una modalidad individual. Estos alumnos presentan rendimientos destacados, ya sea en las unidades curriculares, o en el rendimiento escolar en general. Al parecer, la consistencia de los resultados favorece de igual forma a los alumnos que practican judo asociado a una modalidad colectiva, así como los alumnos que practican judo asociado a la educación física curricular.

La práctica del judo presenta una riqueza pedagógica enorme en relación con el movimiento (en diversos ejes y planos, individual o de grupo, más o menos acelerado), que permite una consecuente evolución en el dominio psicomotor, como argumenta Freire (1991). En la misma línea de pensamiento Fonseca (1988), Nunes (1999) o Picq y Vayer (1988), refieren el movimiento como un factor de gran importancia en el desarrollo del niño. Los mismos autores, consideran de forma general el movimiento como la principal influencia en las primeras formas de pensamiento, aquellas que condicionan la aparición del pensamiento abstracto, porque a través de él (movimiento) es posible adquirir nociones de espacio y tiempo, bases del desarrollo de la inteligencia, y que, consecuentemente, influyen en el aprendizaje de lenguajes simbólicos, particularmente, el lenguaje cualitativo. Estos lenguajes, que contribuyen para un buen rendimiento, tanto a nivel matemático o de la lengua portuguesa, en paralelo a un buen rendimiento académico global. En la misma línea de demostración de los resultados que hemos obtenidos, Molloy, Beerschoten, Borrie, Crilly y Cape (1988), Antunes, Santos, Heredia, Bueno y Mello (2001) y Mazzeo,Cavanagh, Evans, Fiatarone, Hagberg, McAuley y Startzell(1998), indican que la participación en programas de actividad física también tiene beneficios para la función cognitiva, reforzando la teoría de Franklin (2007) y Symons, Cinelli, James y Groff(1997), que alumnos sujetos a elevados programas de actividad física escolar, constatándose efectos positivos en relación con el rendimiento escolar, indicando un aumento de la concentración, una mejoría del cálculo matemático y de la interpretación oral y escrita. Los valores más bajos de rendimiento escolar global y por unidad curricular se centran en los alumnos que apenas realizan educación física, seguidos por orden creciente de los alumnos que frecuentan modalidades colectivas y por los alumnos que no practican ninguna actividad física y deportiva formal. 
Los alumnos que practican judo presentan niveles significativamente superiores en relación con el autoconcepto, concretamente en competencia escolar, aceptación social y comportamiento frente a los alumnos no practicantes.

La práctica continuada del judo promueve un aumento significativo del autoconcepto, concretamente en la aceptación social y la competencia atlética. Los resultados se presentan de acuerdo con Rosa (2008) que indica que los practicantes de artes marciales tienen una imagen positiva ante la opinión pública.

Los responsables de educación y los entrenadores dicen en relación con la competencia escolar, que la práctica de judo aumenta el desarrollo de la concentración y de la capacidad de atención, elevando el autocontrol y la autoconfianza, ayuda en la orientación del estudio y en la definición de las prioridades y objetivos, formando individuos disciplinados cuyo raciocinio e inteligencia se revelan altamente estimulados y provechosos. Simão (2005), dice que la práctica deportiva extracurricular por parte de los alumnos, revela en ellos percepciones más elevadas en las dimensiones de competencia escolar y de aceptación social.

La aceptación social por parte del grupo y modelos sociales en los contextos donde se insiere, se valoriza porque los alumnos que practican judo revelan un perfil de actitud controlada, disciplinada y responsable, con elevada capacidad de adaptación, promoviendo un elevado componente ético de respeto, y un perfil de autoconfianza. Como hace referencia Lima (1988), el deporte se asume también como forma de compensación social, ya que permite a la persona valorizar su ego mediante la afirmación de su personalidad y corporalidad.

En cuanto a la dimensión de competencia atlética, se ha comentado que desarrolla las capacidades físicas, condicionales y coordinativas, así como la tipología morfológica adecuada, aumentando la percepción de autoeficacia y el concepto de corporalidad. En el aspecto físico, el practicante de judo sale valorizado, dado que esta modalidad promueve un desarrollo equilibrado del cuerpo, con una actitud postural equilibrada, factores que contribuyen para un estado de bienestar psicológico. En paralelo, los factores morfológicos positivos y equilibrados y el desarrollo de las capacidades motoras, se han comentado como influencias positivas en esta dimensión y, consecuentemente, en el autoconcepto y bienestar, así como nos afirman Nash (1970), Sastre (1999) o Vasconcelos-Raposo, Teixeira y Gonçalves (2004). 
Los responsables de educación y los entrenadores han referido que el dominio de la conducta sale beneficiado en la medida en que el alumno de judo comparte una cultura ética y de respeto, asumiendo reglas de disciplina, promoviendo una estabilidad emocional y de comportamiento, así como un perfil de actuación basado en la concentración, aspectos que también defienden Castarlenas y Molina (2002).

La práctica del judo ha revelado beneficios pedagógicos en el rendimiento escolar global de los alumnos, evidenciando resultados positivos, aunque no significativos, en el rendimiento escolar cuando se comparan con otros dominios deportivos.

\section{Conclusiones}

De acuerdo con los objetivos de investigación, se han encontrado resultados que evidencian que la práctica de judo presenta una relación positiva con la evolución del autoconcepto, autoestima y rendimiento académico de los alumnos que optan por la práctica de esta modalidad deportiva. Los resultados de las correlaciones obtenidas inducen que las dimensiones que integran la variable de autoconcepto (competencia escolar, aceptación social, competencia atlética, aspecto físico y comportamiento) parecen ejercer esta relación de asociación positiva como nos es referido por Batista y Cubo (2009).

Hattie (1992) señala una influencia positiva del autoconcepto en la competencia escolar y en el comportamiento, así como Oliveira (1999) refiere una relación de influencia del comportamiento en el autoconcepto. La misma relación de asociación positiva se observó en una influencia aparente de las dimensiones de aceptación social, competencia atlética, aspecto físico y comportamiento en la variable autoestima, dimensiones que contribuyen como factores de constitución en el bienestar psicológico, como defienden Sonstroem (1984, 1997),Fox (2000),Biddle y Mutrie (2001), Durkin y Paxton (2002) o Batista y Cubo (2009). Meneses y Simões (2006) apuntan los mismos resultados, aunque estos presenten también en su estudio una relación positiva de la autoestima con las competencias escolares. Las dimensiones del autoconcepto, con excepción de la competencia atlética, no revelaron valores de correlación significativos en la variable rendimiento escolar, así como entre ésta y las variables autoconcepto y autoestima no se verificaron factores de correlación significativos. Estos resultados no 
están de acuerdo totalmente con Holly (1987), que señala factores de relación entre la autoestima y el rendimiento escolar, así como Schunk (1990) observó relaciones positivas entre las competencias escolares de los alumnos y el rendimiento de estos, así como el bajo índice de rendimiento escolar aparece asociado a un bajo autoconcepto (Serra 1986a). Al destacar y tener en consideración, las opiniones de Burns (1979) o Rodrigues (1994 en Serrão, 2001), que señalan que se verifica una bidireccionalidad en la relación entre los resultados escolares y el autoconcepto a lo largo del recorrido escolar. Durante los primeros años escolares, el niño atribuye gran importancia a los éxitos o fracasos que pueda obtener, y la acumulación de estos tendrán gran influencia en la formación del autoconcepto. Posteriormente, el autoconcepto pasa a ser determinante en la forma como el niño enfrenta la escuela, influenciando los resultados escolares.

También se observaron relaciones de asociación positivas entre las dimensiones del autoconcepto en los alumnos que practican judo. De esta forma, la dimensión de competencia escolar presenta valores de relación positivos con la dimensión comportamiento. La dimensión de la aceptación social presenta valores de relación positiva con la competencia atlética, el aspecto físico y el comportamiento. La competencia atlética demostró valores de relación positiva con la aceptación social, el aspecto físico el comportamiento. La dimensión de aspecto físico reveló una relación positiva con las dimensiones de aceptación social y de comportamiento, así como esta última presenta una relación positiva con todas las otras dimensiones, concretamente con el rendimiento escolar, la aceptación social, la competencia atlética y el aspecto físico.

\section{Referencias bibliográficas}

Antunes, H.; Santos, R.; Heredia, R.; Bueno, O. \& Mello, M. (2001). Alterações cognitivas em idosas decorrentes do exercício físico sistematizado. Revista da Soba$m a$, v. 6, p. 27-33, 2001.

Batista, P. (1995). Satisfação com a imagem corporal e auto-estima - Estudo comparativo de adolescentes envolvidas em diferentes niveis de actividade fisica. Dissertação de Doutoramento não publicada, FCDEF - Universidade do Porto, Portugal.

Batista, M. \& Cubo, S. (2011). La Práctica de Judo en Relación con el Autoconcepto, la Autoestima y el Rendimiento Escolar de los Estudiantes de Primer Ciclo de Primaria. Dissertação para obtenção do Grau de Doutor.Badajoz: UNEX. 
La práctica del judo como una modulación del autoconcepto, la autoestima y el rendimiento escolar de los estudiantes de primer ciclo de educación primaria

Batista, M. \& Cubo, S. (2009). Bem Estar Psicológico - Relação entre prática de Judo e Autoconceito. Dissertação para obtenção de Diploma de Estudos Avançados e Suficiência Investigadora.Badajoz: UNEX.

Bento, J. (2004). Desporto para crianças e jovens: das causas e dos fins. In: Gaya, A., Marques, A. \&Tani, G. (Orgs.). Desporto para Crianças e Jovens - Razões e Finalidades (pp.21-28). Porto Alegre: Editora da UFRGS.

Bento, J. (1995). O outro lado do desporto - Vivências e reflexão pedagógicas. Porto: Campo dasLetras - Editores, S. A.

Bourdieu, P. (1979), La Distinction. Critique sociale du jugement. Paris: Les Éditions de Minuit.

Biddle, S. \& Mutrie, N. (2001).Psychology of physical activity: Determiants, Well-being and interventions. London: Routledge.

Burns, R. (1979). The self-concept in theory, measurement and behaviour.London: Longman.

Castarlenas, J. \& Molina, J. (2002). El judo en la educación fisica escolar: unidades didácticas.Barcelona: Hispano Europea.

Carratalá, V. (2012). O Judo Nos JovensCom Risco De Exclusão Social. Actas do congreso europeu de inclusão social através do judo e outrodeportos. Lisboa: ULHT.

Cocke, A. (2002). Brain May Also Pump up from Workout. Retrieved April 11, 03, from Society for Neuroscience Annual Meeting Web Site: http://www.neurosurgery.medsch.ucla.edu/whastnew/societyforneuroscience.htm.

Delgado, N. (2005). Judo.[On line]. Available: http://www.nunodelgado.net/engine. php?cat $=82$ em 23-11-08.

Durkin, S. \& Paxton, S. (2002). Predictors of vulnerability to reduced body image satisfaction and psychological wellbeing in response to exposure to idealized female media images in adolescent girls. Journal of Psychosomatic Research, 53, 995-1005.

Dwyer, T., Coonan, W., Leitch, D., Hetzel, B. \& Baghurst, R. (1983).An investigation of the effects of daily physical activity on the health of primary school students in South Australia.International Journal of Epidemiologists, 12(3), 308-313.

Esculcas, C. (1999). Actividade Física e Práticas de LazernaAdolescênciaPromoção e ManutençãoemFunção da Natureza da Actividade Física e do Estatuto Sócio-económico.Dissertação de Mestrado. Porto: FCDEF - UP

Faustino, A. (1994). Estudo da influência de factores biossociais e capacidades fisicas na evolução da imagem corporal em jovens dos 13 aos 15 anos de idade. Lisboa: FMH.

Fonseca, V. (1988). Da Filogénese à Ontogénese da Motricidade. Porto Alegre: Artes Médicas.

Fox, R. (2000). The effects of exercise on selfperceptions and self-esteem. In Stwart J. H. Biddle, Kenneth R. Fox, \& Stephen H. Boutcher (Eds.), Psysical activity and psychological well-being (pp.88-117). London: Routledge.

Franklin, S. (2007). NCPPA Hopes to Use Strength of Coalition to Introduce Physical Activity into the Reauthorization of the Elementary and Secundary Education Activity in National Coalition for Promoting Physical Activity.URL: http://ncppa.org/\#_ednref4. 
Freire, P. (1991). A Educação na Cidade. São Paulo: Cortez Editora

Gallahue, D. \& Ozmun, C. (2001). Compreendendo o desenvolvimento motor: bebés, crianças, adolescentes e adultos ( $2^{\mathrm{a}}$ ed.). São Paulo: Phorte.

Hattie, J. (1992). Self-Concept.New Jersey: Lawrence Erlbaum Associates.

Holly, W. (1987). Self Esteem: Does it Contribute to Student's Academic Success?. Oregon: School of Study Council, University of Oregon, Eugene.

Lima, J. (1988). Efeito da atividade fisica regular no comportamento da pressão arterial de hipertensos. Dissertação de Mestrado. Santa Maria: Universidade Federal de Santa Maria.

Marivoet, S. (2001). Hábitos desportivos da População Portuguesa. Lisboa: Instituto Nacional de Formação e Estudos do Desporto.

Martins, M., Peixoto, F., Mata, L. \& Monteiro, V. (1995). Escala de Auto-Conceito para Crianças e Pré-Adolescentes de SusanHarter. ProvasPsicológicasem Portugal, $1,78-89$

Mazzeo, R., Cavanagh, P., Evans, W., Fiatarone, M., Hagberg, J, McAuley, E. \&, Startzell J (1998). ACSM position stand: on exercise and physical activity for older adults. Medicin Scientific Sports Exercíce 30:992-1008.

Meneses, R. \& Simões, D. (2006). Preditores da auto-estima global em crianças. Congresso "Exercício, Desporto e Saúde: Sinergias da Psicologia e Medicina", Lisboa.

Molloy, D., Beerschoten, D., Borrie, M., Crilly, R. \& Cape, R. (1988). Acute effects of exercice on neuropsychological function in elderly subjects. Journal of the American Geriatrics Society, 36, 29-33.

Musitu, G.; Román, J. \& Gutiérrez, M. (1996). Educación familiar y socialización de los hijos. Barcelona: Idea Books

Nash, J. (1970). Developmental psychology. Englewood Cliffs: Prentice-Hall, Inc.

Neto, C. (1994). Desporto infantil: A criança e a actividadedesportiva. Horizonte, 10(60), 203-206.

Nunes, M. (1999). Os Grandes Desafios da Autarquia no Âmbito do Desporto, umaProposta de Elaboração de um Plano de DesenvolvimentoDesportivo Municipal. Horizonte, 15 (89), 33-39.

Oliveira, J. (1999). Psicologia da educação: Escola, aluno - aprendizagem. Lisboa: Editorial Presença.

Oliveira, R. (2008). A iniciação do judo naidadepré-escolar e seusbeneficios. [On line]. Available: http://www.judobushido.com.br/conteudo.php?id=17em 25-01-09.

Picq, L. \& Vayer, P. (1988). Educação Psicomotora e Retardo Mental, aplicação aos diferentes tipos de inadaptação. São Paulo: Editora Manole

Queiroz, E. \& Gomes, L. (n.d.). Judo emsuasdimensõesintelectuais, morais e físicas: um componente valioso para o processo de ensino-aprendizagemnaeducação física escolar. [On line]. Available: http://www.judobrasil.com.br/2007/judo_artigo.pdf em 24-10-08.

Rosa, V. (2008a). «As artes marciais e os desportos de combate em números: umolharexploratório sobre os dados numéricos ouestatísticosem Portugal», resumo da proposta de comunicação paraparticipaçãonas X Jornadas do Departamento de Sociologia da Universidade de Évora, 15, 16 e 17de Maio de 2008. 
La práctica del judo como una modulación del autoconcepto, la autoestima y el rendimiento escolar de los estudiantes de primer ciclo de educación primaria

Rosa, V. (2008b).Motivações e entendimentos dos praticantes dos desportos de combate dual: um olharexploratório,: in CDROM ofAbstracts of the 1st European Scientific Congress of Judo "Learning and Performance". Lisbon: UniversidadeLusófona de Humanidades e Tecnologias.

Sastre, M. (1999). Lay conceptions of well-being and rules used in well-being judgements among young, middle-aged, and elderly adults. Social Indicators Research 47, 203-231.

Schunk, D. (1990). Self-concept and school achievement.In Rofers, C. \&Kutnick, P. (Eds.).The socialpsychology of the primary school (pp. 70-79). London: Routledge.

Serra, A. (1986a). O Inventário Clínico do Auto-Conceito. Psiquiatria Clínica, 7(2), 67-84.

Serrão, O. (2001). A relação entre a motivação e o auto-conceito). Importância do estatuto Escolar nos perfismotivacionais de crianças do $4^{\circ}$ ano de escolaridade. Monografia, Instituto Superior de PsicologiaAplicada, Lisboa.

Shephard, R. (1997). Aging, physical activity, and health. Champaign: Human Kinetics Publisher.

Sonstroem, R. (1997). Physical activity and self-esteem.In W. P. Morgan (Ed.) Physical activity and mental health. Washington, D.C.: Taylor and Francis, 127-147.

Sonstroem, R. (1984). Exercise and self-esteem.Exerciseand Sport SciencesReview, 12, 123-155.

Simão, R. (2005). A Relação entre Actividades Extracurriculares e o Desempenho Académico, Motivação, Autoconceito, e Auto-Estima dos alunos. Lisboa: Instituto Superior de Psicologia Aplicada.

Symons, C., Cinelli, B., James, T. \& Groff, P. (1997). Bridging student health risks and academic achievement through comprehensive school health programs. Journal of School Health; 67(6): 220-227; [23].

Tremblay, M., Inman, J. \& Willms, J. (2000). The Relationship Between Physical Activity, Self-Esteem, and Academic Achievement in 12-Year-Old Children. PediatricExerciseScience, 12, 312-324.

Vasconcelos Raposo, J.; Teixeira, C. \&Gonçalves, O. (2004). O Bem estar psicológico em adolescentes: A relação entre prática do exercício físico, auto estima e satisfação corporal. Revista Portuguesa de Ciências do Desporto. Vol 4, n² (44-50).

Yang, X., Telama, R. \& Laakso, L. (1996). Parent`s Physical Activity. Socio-Economic Status and Education as Predictors of Physical and Sport, among Children and Youths: A 12-year Follow-Up Study. International Review for the Sociology of Sport, 31 (3), 94-273. 